\title{
Semiglobal Stabilization via Output-Feedback for a Class of Nontriangular Nonlinear Systems with an Unknown Coefficient
}

\author{
Mengliang Liu, Yungang Liu, and Fengzhong Li \\ School of Control Science and Engineering, Shandong University, Jinan 250061, China \\ Correspondence should be addressed to Yungang Liu; lygfr@sdu.edu.cn
}

Received 27 December 2014; Accepted 12 March 2015

Academic Editor: Ricardo Femat

Copyright (C) 2015 Mengliang Liu et al. This is an open access article distributed under the Creative Commons Attribution License, which permits unrestricted use, distribution, and reproduction in any medium, provided the original work is properly cited.

\begin{abstract}
This paper is devoted to the semiglobal stabilization via output-feedback for a class of uncertain nonlinear systems. Remark that the systems in question contain an unknown control coefficient which inherently depends on the system output and allow largerthan-two order growing unmeasurable states which is the obstruction of global stabilization via output-feedback. By introducing a recursive reduced-order observer and combining with saturated state estimate, a desired output-feedback controller is explicitly constructed for the systems. Under the appropriate choice of design parameters, the controller can make the closed-loop system semiglobally attractive and locally exponentially stable at the origin. A simulation example is provided to illustrate the effectiveness of the proposed approach.
\end{abstract}

\section{Introduction}

In this paper, we consider the semiglobal stabilization via output-feedback for a class of nonlinear systems described by

$$
\begin{gathered}
\dot{x}_{i}=x_{i+1}+\phi_{i}(t, x), \quad i=1, \ldots, n-1, \\
\dot{x}_{n}=g(t, x) u+\phi_{n}(t, x), \\
y=x_{1},
\end{gathered}
$$

where $x=\left[x_{1}, \ldots, x_{n}\right]^{\mathrm{T}}$ is the system state with the initial value $x\left(t_{0}\right)=x_{0} ; u \in \mathbf{R}$ and $y \in \mathbf{R}$ are the input and output of the system, respectively; $\phi_{i}:\left[t_{0},+\infty\right) \times \mathbf{R}^{n} \rightarrow \mathbf{R}, i=1, \ldots, n$, and $g:\left[t_{0},+\infty\right) \times \mathbf{R}^{n} \rightarrow \mathbf{R}$ are unknown but continuous functions, called nonlinearities and control coefficient of the system, respectively. In what follows, suppose that only the output $y$ is measurable.

Rigorously speaking, the control objective of this paper is, for any given constant $\rho_{0}>0$ (may be arbitrarily large), to seek a dynamic output-feedback controller for system (1) as follows:

$$
\begin{gathered}
\dot{\eta}=\psi(\eta, y), \quad \eta \in \mathbf{R}^{n-1}, \\
u=\varphi(\eta, y),
\end{gathered}
$$

where $\psi(\cdot)$ and $\varphi(\cdot)$ are continuous functions satisfying $\psi(0,0)=0$ and $\varphi(0,0)=0$, such that the closed-loop system is

(i) semiglobally attractive; that is, by starting from the compact set $\Gamma_{\rho_{0}}^{x} \times \Gamma_{\rho_{0}}^{\eta}$, all the trajectories of the closedloop system converge to the origin, where $\Gamma_{\rho_{0}}^{x}=\{x \mid$ $\left.x \in\left[-\rho_{0}, \rho_{0}\right] \times \cdots \times\left[-\rho_{0}, \rho_{0}\right] \subset \mathbf{R}^{n}\right\}$ and $\Gamma_{\rho_{0}}^{\eta}=\{\eta \mid \eta \epsilon$ $\left.\left[-\rho_{0}, \rho_{0}\right] \times \cdots \times\left[-\rho_{0}, \rho_{0}\right] \subset \mathbf{R}^{n-1}\right\}$

(ii) locally exponentially stable; that is, the closed-loop system is locally exponentially stable at the origin $(x, \eta)=(0,0)$.

As in $[1,2]$, we introduce the dilation $\delta_{\varepsilon}(x)=$ $\left(x_{1}, \varepsilon x_{2}, \ldots, \varepsilon^{n-1} x_{n}\right)$ with $\varepsilon$ being any positive constant. Based on this, the following assumptions are made on the nonlinearities and the control coefficient of system (1).

Assumption 1. There exist known positive continuous functions $g_{0}(y)$ and $g_{1}(y)$ such that, for any $\varepsilon \geq 1$,

$$
g_{0}(y) \leq g\left(t, \delta_{\varepsilon}(x)\right) \leq g_{1}(y) .
$$


Assumption 2. There exist a known constant $\sigma \in[0,1)$ and known nonnegative continuous function $c_{i}(x), i=1, \ldots, n$, such that, for any $\varepsilon \geq 1$,

$$
\phi_{i}\left(t, \delta_{\varepsilon}(x)\right) \leq \varepsilon^{i-1+\sigma} c_{i}(x)\left(\left|x_{1}\right|+\cdots+\left|x_{n}\right|\right), \quad i=1, \ldots, n .
$$

For nonlinear systems, semiglobal stabilization via output-feedback has attracted a great deal of attention (see, e.g., [3-13] and references therein), since it has a control objective meeting the needs of practical application and, compared to the global case, it requires rather weak restrictions on the system nonlinearities. We would like to stress that our problem is nontrivial and rather difficult to solve, which is mainly due to the generality of Assumptions 1 and 2 . On the one hand, system (1) allows additional unknowns and nonlinearities in the control coefficient. Assumption 1 shows that the control coefficient of system (1) is unknown and inherently depends on the system output, essentially different from [7-10, 14-16] where the control coefficients are known/unknown constants or known functions of the system output. On the other hand, system (1) can be of nontriangular structure and has inherent nonlinearities. Assumption 2 indicates that, for any $i=1, \ldots, n, \phi_{i}(\cdot)$ inherently depends on all the states, rather than merely on $x_{1}, \ldots, x_{i}$ like $[8,10,11]$. Also, due to the presence of $c_{i}(\cdot)$ 's, system (1) allows more serious nonlinearities than those in $[10,11,17]$. Particularly, in [10], the system nonlinearities $\psi_{i}(\cdot)$ 's are required to satisfy

$$
\begin{array}{r}
\left|\psi_{i}(t, \eta, u)\right| \leq \mu_{\psi_{i}}(y) \sum_{j=1}^{i}\left|\eta_{j}\right|+\nu_{\psi_{i}}(y) \sum_{j=2}^{i-1}\left|\eta_{j}\right|^{(i-1) /(j-1)} \\
i=1, \ldots, n,
\end{array}
$$

where $\mu_{\psi_{i}}(\cdot), i=1, \ldots, n$, and $\nu_{\psi_{i}}(\cdot), i=3, \ldots, n$, are known nonnegative smooth functions and $\nu_{\psi_{1}}(\cdot)=v_{\psi_{2}}(\cdot) \equiv 0$. This is a special case of Assumption 2 with $c_{i}(x) \geq \mu_{\psi_{i}}(y)+$ $v_{\psi_{i}}(y) \sum_{j=1}^{i}\left|x_{j}\right|^{(i-1) /(j-1)-1}, i=1, \ldots, n$. Therefore, one can see that the nonlinearities $\phi_{i}(\cdot)$ 's of system (1) permit largerthan-two order growing unmeasurable states, which is the obstruction of global stabilization via output-feedback.

In this paper, a semiglobal stabilization scheme via output-feedback is proposed for uncertain nontriangular nonlinear system (1) with serious nonlinearities and the unknown control coefficient depending on the system output. Specifically, a state-feedback controller is first constructed for a nominal system (where $\phi_{i}(\cdot) \equiv 0, i=1, \ldots, n$ ), which ensures that the closed-loop system (corresponding to the nominal system) is globally exponentially stable. Then, a recursive reduced-order observer is introduced to recover the unmeasurable states of system (1). Based on these and combining with saturated state estimate $[3,4]$, a semiglobal output-feedback stabilizer is explicitly constructed for system (1). By appropriately choosing design parameters, the controller can guarantee that the closed-loop system (corresponding to system (1)) is semiglobally attractive and locally exponentially stable at the origin.

The remainder of this paper is organized as follows. Section 2 presents semiglobal output-feedback control design for system (1). Section 3 provides the main results and the rigorous performance analysis of the closed-loop system. Section 4 gives a numerical example to illustrate effectiveness of the proposed method. Section 5 addresses some concluding remarks. This paper ends with an appendix that collects two proofs of important propositions.

\section{Semiglobal Output-Feedback Control}

The section is to design a semiglobal stabilizer via outputfeedback for system (1) under Assumptions 1 and 2.

To achieve this, we introduce the coordinate transformation

$$
\begin{gathered}
z_{i}=\frac{x_{i}}{L^{i-1}}, \quad i=1, \ldots, n, \\
v=\frac{u}{L^{n}},
\end{gathered}
$$

which changes system (1) into the following:

$$
\begin{gathered}
\dot{z}_{i}=L z_{i+1}+\frac{\phi_{i}\left(t, \delta_{L}(z)\right)}{L^{i-1}}, \quad i=1, \ldots, n-1, \\
\dot{z}_{n}=\operatorname{Lg}\left(t, \delta_{L}(z)\right) v+\frac{\phi_{n}\left(t, \delta_{L}(z)\right)}{L^{n-1}}, \\
y=z_{1},
\end{gathered}
$$

where $z=\left[z_{1}, \ldots, z_{n}\right]^{\mathrm{T}}$ and $L \geq 1$ is a design parameter to be determined later.

For simplicity, we denote $\widetilde{g}(t, z)=g\left(t, \delta_{L}(z)\right)$ and $\widetilde{\phi}_{i}(t, z)=\phi_{i}\left(t, \delta_{L}(z)\right) / L^{i-1}, i=1, \ldots, n$. By Assumption 2, it can be verified that, for $i=1, \ldots, n$,

$$
\tilde{\phi}_{i}(t, z) \leq L^{\sigma} c_{i}(z)\left(\left|z_{1}\right|+\cdots+\left|z_{n}\right|\right) .
$$

Moreover, it is worth stressing that the semiglobal stabilization of system (1) is implied by that of system (7). In the sequel, we turn to the controller design of system (7).

We first establish the following proposition, which gives a state-feedback controller for system (7) without considering the nonlinearities $\widetilde{\phi}_{i}(\cdot)$ 's.

Proposition 3. Under Assumption 1, consider the following nominal system:

$$
\begin{gathered}
\dot{z}_{i}=L z_{i+1}, \quad i=1, \ldots, n-1, \\
\dot{z}_{n}=L \widetilde{g}(t, z) \widetilde{v} .
\end{gathered}
$$

There exist positive constants $k_{i}, i=1, \ldots, n$, such that system (9) is globally exponentially stabilized by the state-feedback controller:

$$
\widetilde{v}(z)=-\frac{k_{n}}{g_{0}\left(z_{1}\right)}\left(z_{n}+k_{n-1} z_{n-1}+\cdots+k_{1} z_{1}\right) .
$$

Proof. Choose constants $k_{i}, i=1, \ldots, n-1$, such that polynomial $p(s)=s^{n-1}+k_{n-1} s^{n-2}+\cdots+k_{2} s+k_{1}$ is Hurwitz. Then, we rewrite system (9) as

$$
\begin{gathered}
\dot{z}_{[n-1]}=L\left(A_{1}-B_{1} K_{1}\right) z_{[n-1]}+L B_{1}\left(z_{n}+K_{1} z_{[n-1]}\right), \\
\dot{z}_{n}=L \tilde{g}(t, z) \widetilde{v},
\end{gathered}
$$


where $z_{[n-1]}=\left[z_{1}, \ldots, z_{n-1}\right]^{\mathrm{T}}, K_{1}=\left[k_{1}, \ldots, k_{n-1}\right]$,

$$
A_{1}=\left[\begin{array}{cccc}
0 & 1 & \cdots & 0 \\
\vdots & \vdots & \ddots & \vdots \\
0 & 0 & \cdots & 1 \\
0 & 0 & \cdots & 0
\end{array}\right] \in \mathbf{R}^{(n-1) \times(n-1)}
$$

$$
B_{1}=\left[\begin{array}{c}
0 \\
\vdots \\
0 \\
1
\end{array}\right] \in \mathbf{R}^{(n-1) \times 1} \text {. }
$$

Noting that $A_{1}-B_{1} K_{1}$ is a Hurwitz matrix, there exists a positive definite matrix such that

$$
\left(A_{1}-B_{1} K_{1}\right)^{\mathrm{T}} P_{1}+P_{1}\left(A_{1}-B_{1} K_{1}\right) \leq-2 I_{n-1} .
$$

Then, we define the Lyapunov function

$$
V_{z}(z)=z_{[n-1]}^{\mathrm{T}} P_{1} z_{[n-1]}+\frac{\left(z_{n}+K_{1} z_{[n-1]}\right)^{2}}{2}
$$

whose derivative along system (11) is as follows:

$$
\begin{aligned}
\dot{V}_{z}(z) \leq & -2 L\left\|z_{[n-1]}\right\|^{2}+2 L z_{[n-1]}^{\mathrm{T}} P_{1} B_{1}\left(z_{n}+K_{1} z_{[n-1]}\right) \\
& +L\left(z_{n}+K_{1} z_{[n-1]}\right) \tilde{g}(t, z) \widetilde{v} \\
& +\left(z_{n}+K_{1} z_{[n-1]}\right) \\
& \cdot K_{1} L\left(\left(A_{1}-B_{1} K_{1}\right) z_{[n-1]}+B_{1}\left(z_{n}+K_{1} z_{[n-1]}\right)\right) \\
\leq & -2 L\left\|z_{[n-1]}\right\|^{2}+L\left(2\left\|P_{1} B_{1}\right\|+\left\|K_{1}\left(A_{1}-B_{1} K_{1}\right)\right\|\right) \\
& \cdot\left\|z_{[n-1]}\right\|\left|z_{n}+K_{1} z_{[n-1]}\right|+L k_{n-1}\left(z_{n}+K_{1} z_{[n-1]}\right)^{2} \\
& +L \tilde{g}(t, z)\left(z_{n}+K_{1} z_{[n-1]}\right) \widetilde{v} .
\end{aligned}
$$

Using Young's inequality, we have

$$
\begin{aligned}
& \left(2\left\|P_{1} B_{1}\right\|+\left\|K_{1}\left(A_{1}-B_{1} K_{1}\right)\right\|\right)\left\|z_{[n-1]}\right\|\left|z_{n}+K_{1} z_{[n-1]}\right| \\
& \leq\left\|z_{[n-1]}\right\|^{2}+C\left|z_{n}+K_{1} z_{[n-1]}\right|^{2},
\end{aligned}
$$

where $C=\left(2\left\|P_{1} B_{1}\right\|+\left\|K_{1}\left(A_{1}-B_{1} K_{1}\right)\right\|\right)^{2} / 4$. Substituting the above estimation into (15) yields

$$
\begin{aligned}
\dot{V}_{z}(z) \leq & -L\left\|z_{[n-1]}\right\|^{2}+L\left(k_{n-1}+C\right)\left(z_{n}+K_{1} z_{[n-1]}\right)^{2} \\
& +L \tilde{g}(t, z)\left(z_{n}+K_{1} z_{[n-1]}\right) \widetilde{v}
\end{aligned}
$$

Choose $k_{n} \geq k_{n-1}+C+1$ and construct the state feedback controller

$$
\widetilde{v}(z)=-\frac{k_{n}}{g_{0}\left(z_{1}\right)}\left(z_{n}+K_{1} z_{[n-1]}\right) .
$$

Then, by Assumption 1, we derive

$$
\dot{V}_{z}(z) \leq-L\left\|z_{[n-1]}\right\|^{2}-L\left(z_{n}+K_{1} z_{[n-1]}\right)^{2},
$$

which, together with (14), implies that the closed-loop system consisting of (9) and (10) is globally exponentially stable.

This completes the proof.

Based on controller (10) of nominal system (9), we construct a desired output-feedback controller for system (7). Motivated by $[8,18,19]$, we introduce the following recursive reduced-order observer to recover unmeasurable states $z_{i}$, $i=2, \ldots, n$, of system (7):

$$
\begin{array}{cc}
\dot{\eta}_{2}=-L\left(\ell_{2} \eta_{2}+\ell_{2}^{2} y\right), & \widehat{z}_{2}=\eta_{2}+\ell_{2} \widehat{z}_{1}, \\
\dot{\eta}_{3}=-L\left(\ell_{3} \eta_{3}+\ell_{3}^{2} \widehat{z}_{2}\right), & \widehat{z}_{3}=\eta_{3}+\ell_{3} \widehat{z}_{2}, \\
\vdots & \vdots \\
\dot{\eta}_{n}=-L\left(\ell_{n} \eta_{n}+\ell_{n}^{2} \widehat{z}_{n-1}\right), & \widehat{z}_{n}=\eta_{n}+\ell_{n} \widehat{z}_{n-1},
\end{array}
$$

where $\widehat{z}_{1}=z_{1}$ and $\ell_{i}>1, i=2, \ldots, n$, are the gains to be determined later. For the later use, we denote $\widehat{z}=\left[\widehat{z}_{1}, \ldots, \widehat{z}_{n}\right]^{\mathrm{T}}$ and $\eta=\left[\eta_{2}, \ldots, \eta_{n}\right]^{\mathrm{T}}$.

Furthermore, using saturated state estimate, we construct the following output-feedback controller for system (7):

$$
\begin{aligned}
v(\widehat{z})=-\frac{k_{n}}{g_{0}\left(\widehat{z}_{1}\right)}( & \text { sat }\left(\widehat{z}_{n}\right)+k_{n-1} \operatorname{sat}\left(\widehat{z}_{n-1}\right) \\
& \left.+\cdots+k_{2} \operatorname{sat}\left(\widehat{z}_{2}\right)+k_{1} \widehat{z}_{1}\right),
\end{aligned}
$$

where $k_{i}, i=1, \ldots, n$, are the same as those in Proposition 3; the saturation function sat $: \mathbf{R} \rightarrow[-M, M]$ is defined as

$$
\begin{aligned}
& \text { sat }(a)= \begin{cases}-M, & a<-M, \\
a, & |a| \leq M, \\
M, & a>M,\end{cases} \\
& M \geq \max _{z \in \Omega_{z}}\|z\|_{\infty},
\end{aligned}
$$

with $\Omega_{z}=\left\{z \in \mathbf{R}^{n} \mid V_{z}(z) \leq 1+\max _{z \in \Gamma_{\rho_{0}}^{z}} V_{z}(z)\right\}, \Gamma_{\rho_{0}}^{z}=\{z \mid$ $\left.z \in\left[-\rho_{0}, \rho_{0}\right] \times \cdots \times\left[-\rho_{0}, \rho_{0}\right] \subset \mathbf{R}^{n}\right\}$, and $\|z\|_{\infty}$ standing for the $\infty$-norm of vector $z$.

\section{Main Results}

This section is devoted to the performance analysis of the closed-loop system consisting of (7), (20), and (21) and summarizes the main results of this paper.

Define $e_{i}=z_{i}-\ell_{i} z_{i-1}-\eta_{i}, i=2, \ldots, n$, and denote $e=$ $\left[e_{2}, \ldots, e_{n}\right]^{\mathrm{T}}$. Then, by (7) and (20), we deduce

$$
\begin{aligned}
\dot{e}_{i}= & L z_{i+1}+\widetilde{\phi}_{i}(\cdot)-L \ell_{i} e_{i}-\ell_{i} \widetilde{\phi}_{i-1}(\cdot) \\
& -L \ell_{i}^{2}\left(z_{i-1}-\widehat{z}_{i-1}\right), \quad i=2, \ldots, n-1, \\
\dot{e}_{n}= & L \tilde{g}(t, z) v(\widehat{z})+\widetilde{\phi}_{n}(\cdot)-L \ell_{n} e_{n}-\ell_{n} \widetilde{\phi}_{n-1}(\cdot) \\
& -L \ell_{n}^{2}\left(z_{n-1}-\widehat{z}_{n-1}\right) .
\end{aligned}
$$


Define the Lyapunov function $V_{e}(e)=\sum_{i=2}^{n}\left(e_{i}^{2} / 2\right)$ and choose the same Lyapunov function $V_{z}(z)$ used in Proposition 3. On the set $\Gamma_{M}^{z} \times \mathbf{R}^{n-1}=\{(z, \eta) \mid z \in[-M, M] \times$ $\left.\cdots \times[-M, M] \subset \mathbf{R}^{n}, \eta \in \mathbf{R}^{n-1}\right\}$, there hold Propositions 4 and 5 (whose detailed proofs are given in Appendices A and $\mathrm{B}$, resp.), which will play a key role in the later performance analysis.

Proposition 4. For system (7) on the set $\Gamma_{M}^{z} \times \mathbf{R}^{n-1}$, there exist constants $c>0$ and $d_{1}>0$, such that

$$
\begin{aligned}
\left.\dot{V}_{z}(z)\right|_{\Gamma_{M}^{z} \times \mathbf{R}^{n-1}} & \\
\leq & -\frac{c L}{2}\|z\|^{2}+L^{\sigma} d_{1}\|z\|^{2} \\
& +L \min \left\{\widehat{\alpha}_{n} e_{n}^{2}+\sum_{i=2}^{n-1} \widehat{\alpha}_{i}\left(\ell_{i+1}, \ldots, \ell_{n}\right) e_{i}^{2}, \widehat{\alpha}_{n}\right\},
\end{aligned}
$$

where $\widehat{\alpha}_{n} \geq 1$ is a constant independent of $\ell_{i}$ 's and $\widehat{\alpha}_{i}\left(\ell_{i+1}\right.$, $\left.\ldots, \ell_{n}\right) \geq \widehat{\alpha}_{n}, i=2, \ldots, n-1$, are certain polynomial functions of their arguments.

Proposition 5. For system (24) on the set $\Gamma_{M}^{z} \times \mathbf{R}^{n-1}$ and constant $c>0$ given in Proposition 4, there holds

$$
\begin{aligned}
\left.\dot{V}_{e}(e)\right|_{\Gamma_{M}^{z} \times \mathbf{R}^{n-1}} \leq & \frac{c L}{4}\|z\|^{2}-L\left(e_{n}-\widetilde{\alpha}_{n}-\frac{2}{c}\right) e_{n}^{2} \\
& -L \sum_{i=2}^{n-1}\left(\ell_{i}-\widetilde{\alpha}_{i}\left(\ell_{i+1}, \ldots, \ell_{n}\right)-\frac{2}{c}\right) e_{i}^{2} \\
& +\sum_{i=2}^{n}\left|e_{i}\right|\left|\widetilde{\phi}_{i}(\cdot)-\ell_{i} \widetilde{\phi}_{i-1}(\cdot)\right|
\end{aligned}
$$

where $\widetilde{\alpha}_{n}>0$ is a constant independent of $\ell_{i}$ 's and $\widetilde{\alpha}_{i}\left(\ell_{i+1}\right.$, $\left.\ldots, \ell_{n}\right)>0, i=2, \ldots, n-1$, are certain polynomial functions of their arguments.

In view of (25) and (26), we recursively determine the observer gains $\ell_{i}$ 's as follows:

$$
\begin{gathered}
\ell_{n}(\ell)=\ell \widehat{\alpha}_{n}+\widetilde{\alpha}_{n}+\frac{2}{c}, \\
\ell_{i}(\ell)=\ell \widehat{\alpha}_{i}\left(\ell_{i+1}, \ldots, \ell_{n}\right)+\widetilde{\alpha}_{i}\left(\ell_{i+1}, \ldots, \ell_{n}\right)+\frac{2}{c}, \\
i=n-1, \ldots, 2,
\end{gathered}
$$

such that

$$
\dot{V}_{e}(e) \leq \frac{c L}{4}\|z\|^{2}-L \ell W_{e}(e)+\sum_{i=2}^{n}\left|e_{i}\right|\left|\widetilde{\phi}_{i}(\cdot)-\ell_{i} \widetilde{\phi}_{i-1}(\cdot)\right|,
$$

where $\ell$ is a positive constant to be determined later and $W_{e}(e)=\widehat{\alpha}_{n} e_{n}^{2}+\sum_{i=2}^{n-1} \widehat{\alpha}_{i}\left(\ell_{i+1}, \ldots, \ell_{n}\right) e_{i}^{2}$.

Now, we are ready to address the main results of this paper, which are summarized in the following theorem.
Theorem 6. Consider system (1) under Assumptions 1 and 2. For any given constant $\rho_{0}>0$ (may be arbitrarily large), there exist appropriate $L$ and $\ell_{i}, i=2, \ldots, n$, depending on $\rho_{0}$, such that the closed-loop system, consisting of (1), (20), and (21), is locally exponentially stable, and, by starting from the given compact set $\Gamma_{\rho_{0}}^{x} \times \Gamma_{\rho_{0}}^{\eta}$, all the trajectories of the closed-loop system converge to the origin.

Proof. Motivated by [5, 6, 13], for the closed-loop system consisting of (7), (20), and (21), we choose Lyapunov function

$$
V_{z, \eta}(z, \eta)=V_{z}(z)+\frac{\ln \left(1+V_{e}(e)\right)}{\ln (1+\mu(\ell))},
$$

where $\mu(\ell)=(1 / 2) \sum_{i=2}^{n}\left(2 \rho_{0}+\ell_{i}(\ell) \rho_{0}\right)^{2} \geq \max _{\Gamma_{\rho_{0}}^{z} \times \Gamma_{\rho_{0}}^{\eta}} V_{e}(e)$. From the definitions of $V_{z}(z)$ and $V_{e}(e)$, we see that $V_{z, \eta}(z, \eta)$ is positive definite with respect to $(z, \eta)$. Moreover, by (25) and (28), we obtain that, on the set $\Gamma_{M}^{z} \times \mathbf{R}^{n-1}$,

$$
\begin{aligned}
\left.\dot{V}_{z, \eta}(z, \eta)\right|_{\Gamma_{M}^{z} \times \mathbf{R}^{n-1}} \leq & -\frac{c L}{2}\|z\|^{2}+L^{\sigma} d_{1}\|z\|^{2} \\
& +L \min \left\{W_{e}(e), \widehat{\alpha}_{n}\right\} \\
& +\frac{L}{\ln (1+\mu(\ell))\left(1+V_{e}(e)\right)} \\
& \cdot\left(\frac{c}{4}\|z\|^{2}-\ell W_{e}(e)\right) \\
& +\frac{1}{\ln (1+\mu(\ell))\left(1+V_{e}(e)\right)} \\
& \cdot \sum_{i=2}^{n}\left|e_{i}\right|\left|\widetilde{\phi}_{i}(\cdot)-\ell_{i} \widetilde{\phi}_{i-1}(\cdot)\right| .
\end{aligned}
$$

From $\widehat{\alpha}_{i}(\cdot) \geq \widehat{\alpha}_{n} \geq 1, i=2, \ldots, n-1$, it follows that

$$
\begin{aligned}
\frac{W_{e}(e)}{1+V_{e}(e)} & \geq \frac{\widehat{\alpha}_{n}\left(W_{e}(e) / \widehat{\alpha}_{n}\right)}{1+W_{e}(e) / \widehat{\alpha}_{n}} \geq \frac{\widehat{\alpha}_{n}}{2} \min \left\{\frac{W_{e}(e)}{\widehat{\alpha}_{n}}, 1\right\} \\
& \geq \frac{1}{2} \min \left\{W_{e}(e), \widehat{\alpha}_{n}\right\},
\end{aligned}
$$

which implies that, on the set $\Gamma_{M}^{z} \times \mathbf{R}^{n-1}$,

$$
\begin{aligned}
\left.\dot{V}_{z, \eta}(z, \eta)\right|_{\Gamma_{M}^{z} \times \mathbf{R}^{n-1}} \leq & -\frac{c L}{2}\|z\|^{2}+L^{\sigma} d_{1}\|z\|^{2} \\
& +\frac{c L}{4 \ln (1+\mu(\ell))}\|z\|^{2} \\
& -L\left(\frac{\ell}{2 \ln (1+\mu(\ell))}-1\right) \\
& \cdot \min \left\{W_{e}(e), \widehat{\alpha}_{n}\right\} \\
& +\frac{1}{\ln (1+\mu(\ell))} \\
& \cdot \sum_{i=2}^{n}\left|e_{i}\right|\left|\widetilde{\phi}_{i}(\cdot)-\ell_{i} \widetilde{\phi}_{i-1}(\cdot)\right| .
\end{aligned}
$$


From the choice of $\ell_{i}(\ell)$ 's, we see that $\ell_{i}(\ell)$ 's are polynomial functions of $\ell$. Then, we can find $\ell>1$ to be sufficiently large such that

$$
\begin{gathered}
\frac{\ell}{2 \ln (1+\mu(\ell))}>2, \\
\ln (1+\mu(\ell))>1,
\end{gathered}
$$

which, together with (32), implies that, on the set $\Gamma_{M}^{z} \times \mathbf{R}^{n-1}$,

$$
\begin{aligned}
\left.\dot{V}_{z, \eta}(z, \eta)\right|_{\Gamma_{M}^{z} \times \mathbf{R}^{n-1}} & \\
\leq & -\frac{c L}{4}\|z\|^{2}+L^{\sigma} d_{1}\|z\|^{2}-L \min \left\{W_{e}(e), \widehat{\alpha}_{n}\right\} \\
& +\sum_{i=2}^{n}\left|e_{i}\right|\left|\widetilde{\phi}_{i}(\cdot)-\ell_{i}(\ell) \widetilde{\phi}_{i-1}(\cdot)\right| .
\end{aligned}
$$

Moreover, define the compact set $\Omega=\left\{(z, \eta) \mid V_{z, \eta}(z\right.$, $\left.\eta) \leq 1+\max _{z \in \Gamma_{\rho_{0}}^{z}} V_{z}(z)\right\}$. Then, it is easy to verify the following relation:

$$
\Gamma_{\rho_{0}}^{z} \times \Gamma_{\rho_{0}}^{\eta} \subset \Omega \subset \Gamma_{M}^{z} \times \mathbf{R}^{n-1} .
$$

We now estimate the last term in the right-hand side of (34) on the compact set $\Omega$. By (8) and Young's inequality and noting the boundedness of $c_{i}(z)$ 's and $W_{e}(e)$ on $\Omega$, there exist positive constants $d_{2}$ and $d_{3}$ depending on $\ell_{i}$ 's, such that, on the set $\Omega$,

$$
\begin{aligned}
& \sum_{i=2}^{n}\left|e_{i}\right|\left|\tilde{\phi}_{i}(\cdot)-\ell_{i}(\ell) \tilde{\phi}_{i-1}(\cdot)\right| \\
& \quad \leq L^{\sigma} \sum_{i=2}^{n}\left|c_{i}(z)-\ell_{i}(\ell) c_{i-1}(z)\right|\left|e_{i}\right| \sum_{j=1}^{n}\left|z_{j}\right| \\
& \leq L^{\sigma} d_{2}\|z\|^{2}+L^{\sigma} d_{3} \min \left\{W_{e}(e), \widehat{\alpha}_{n}\right\} .
\end{aligned}
$$

Substituting this into (34), we obtain that, on the set $\Omega$,

$$
\begin{aligned}
\left.\dot{V}_{z, \eta}(z, \eta)\right|_{\Omega} \leq & -\left(\frac{c L}{4}-L^{\sigma} d_{1}-L^{\sigma} d_{2}\right)\|z\|^{2} \\
& -\left(L-L^{\sigma} d_{3}\right) \min \left\{W_{e}(e), \hat{\alpha}_{n}\right\} .
\end{aligned}
$$

By $0 \leq \sigma<1$, we can choose $L \geq 1$ satisfying

$$
L^{1-\sigma} \geq \max \left\{\frac{d_{1}+d_{2}+1}{4 c}, d_{3}+1\right\} .
$$

Then, we obtain that, on the set $\Omega$,

$$
\left.\dot{V}_{z, \eta}(z, \eta)\right|_{\Omega} \leq-L^{\sigma}\|z\|^{2}-L^{\sigma} \min \left\{W_{e}(e), \widehat{\alpha}_{n}\right\} .
$$

By the definitions of $\eta_{i}$ 's and $W_{e}(e)$, we derive $\dot{V}_{z, \eta}(z, \eta)<0$ on $\Omega \backslash\{(0,0)\}$, which implies that, by starting from $\Gamma_{\rho_{0}}^{z} \times \Gamma_{\rho_{0}}^{\eta}$, $(z(t), \eta(t))$ remains in the compact set $\Omega$, and

$$
\lim _{t \rightarrow+\infty}(z(t), \eta(t))=(0,0) .
$$

By $W_{e}(0)=0$ and the continuity of $W_{e}(e)$ on $\Omega$, there exists a neighborhood $\Omega_{0} \subseteq \Omega$ of the origin, such that $W_{e}(e) \leq$ $\widehat{\alpha}_{n}$ for all $(z, \eta) \in \Omega_{0}$. Then, by (39), we obtain that, on the set $\Omega_{0}$,

$$
\left.\dot{V}_{z, \eta}(z, \eta)\right|_{\Omega_{0}} \leq-L^{\sigma}\|z\|^{2}-L^{\sigma} W_{e}(e)
$$

By the definition of $V_{e}(e)$ and $W_{e}(e)$, we deduce that, on the set $\Omega_{0}$,

$$
W_{e}(e) \geq \mu_{1} V_{e}(e) \geq \mu_{2} \frac{\ln \left(1+V_{e}(e)\right)}{\ln (1+\mu(\ell))}
$$

with some positive constants $\mu_{1}$ and $\mu_{2}$. Substituting this into (41) and noting the definitions of $V_{z}(z)$, we have that, on the set $\Omega_{0}$,

$$
\left.\dot{V}_{z, \eta}(z, \eta)\right|_{\Omega_{0}} \leq-L^{\sigma} \mu_{3} V_{z, \eta}(z, \eta)
$$

with positive constant $\mu_{3}$. Therefore, the closed-loop system consisting of (7), (20), and (21) is locally exponentially stable.

By (6), (35) and noting $L \geq 1$, there holds

$$
x \in \Gamma_{\rho_{0}}^{x}, \quad \eta \in \Gamma_{\rho_{0}}^{\eta} \Longrightarrow z \in \Gamma_{\rho_{0}}^{z}, \quad \eta \in \Gamma_{\rho_{0}}^{\eta} \Longrightarrow(z, \eta) \in \Omega .
$$

Furthermore, by the invertibility of (6), we arrive at the fact that the closed-loop system, consisting of (1), (20), and (21), is locally exponentially stable, and, by starting from $\Gamma_{\rho_{0}}^{x} \times \Gamma_{\rho_{0}}^{\eta}$, the trajectories $(x(t), \eta(t))$ converge to the origin.

This completes the proof.

\section{A Simulation Example}

In this section, we consider the following nonlinear system to illustrate the correctness and effectiveness of the theoretical results:

$$
\begin{gathered}
\dot{x}_{1}=x_{2}+x_{1}^{2 / 3} x_{3}^{1 / 3}, \\
\dot{x}_{2}=x_{3}+x_{1} \ln \left(1+x_{2}^{2}\right), \\
\dot{x}_{3}=g(t, x) u+x_{1}, \\
y=x_{1},
\end{gathered}
$$

with $2\left(x_{1}^{2}+1\right) \leq g(t, x) \leq 4\left(x_{1}^{2}+1\right)$. It can be verified that system (45) satisfies Assumption 2 with $\sigma=2 / 3, c_{1}(x)=1$, and $c_{2}(x)=2\left(1+x_{2}^{2}\right)$.

According to the design procedure of the state feedback controller, we choose $k_{1}=0.8$ and $k_{2}=1.2$ and then, by solving the matrix inequality (13), yield

$$
P_{1}=\left(\begin{array}{cc}
1.6875 & -0.5 \\
-0.5 & 0.75
\end{array}\right) \text {. }
$$

Furthermore, by Proposition 3, we choose $k_{3}=5$. 
Let $\rho_{0}=3$. Then, by the above $P_{1},(14)$, and (23), we choose $M=6$. Moreover, from (33) and (38), we choose $\ell=1.5$ and $L=2$ and, in terms of (27), recursively determine $\ell_{2}=10$ and $\ell_{3}=8$. Thus, by the design procedure in Section 2, we construct the following observer:

$$
\begin{array}{cc}
\dot{\eta}_{2}=-2\left(10 \eta_{2}+100 y\right), & \widehat{z}_{2}=\eta_{2}+10 \widehat{z}_{1}, \\
\dot{\eta}_{3}=-2\left(8 \eta_{3}+64 \widehat{z}_{2}\right), & \widehat{z}_{3}=\eta_{3}+8 \widehat{z}_{2},
\end{array}
$$

and design the following output-feedback controller:

$$
v(\widehat{z})=-\frac{5}{2\left(\widehat{z}_{1}^{2}+1\right)}\left(\mathbf{s a t}\left(\widehat{z}_{3}\right)+1.2 \mathbf{s a t}\left(\widehat{z}_{2}\right)+0.8 \widehat{z}_{1}\right) .
$$

Let $g(t, x)=\left(3-\sin x_{2}\right)\left(x_{1}^{2}+1\right)$, and let the initial values of the states be $x(0)=[1,-0.5,-0.5]^{\mathrm{T}}$ and $\eta(0)=$ $[1,-1]^{\mathrm{T}}$. Using MATLAB, Figures $1-3$ are obtained to exhibit the trajectories of the closed-loop system states and the controller, from which the effectiveness of the design method is indeed demonstrated.

\section{Concluding Remarks}

In this paper, the semiglobal stabilization via output-feedback has been investigated for a class of uncertain nontriangular nonlinear systems. Essentially different from the existing related works, the control coefficient of the system is unknown and inherently depends on the system output, and, hence, the scope of the nonlinear systems is considerably broadened. By introducing a recursive reduced-order observer and combining with saturated state estimate, a semiglobal output-feedback controller has been constructed for the uncertain system. Under the appropriate choice of design parameters, the controller can guarantee that the closed-loop system achieves semiglobal attractivity and locally exponential stability. Along this direction, another interesting research problem is how to design a semiglobal finite-time stabilizer via output-feedback for system (1).

\section{Appendices}

The appendix provides the rigorous proofs of Propositions 4 and 5 , which are collected here for the sake of compactness.

\section{A. The Proof of Proposition 4}

By (7) and (19), we have

$$
\begin{aligned}
\dot{V}_{z}(z) \leq & -L\left\|z_{[n-1]}\right\|^{2}-L\left(z_{n}+K_{1} z_{[n-1]}\right)^{2} \\
& +L \widetilde{g}(t, z)\left|z_{n}+K_{1} z_{[n-1]}\right||v(\widehat{z})-\widetilde{v}(z)| \\
& +\left|z_{[n-1]}^{\mathrm{T}} P_{1} \widetilde{\phi}_{[n-1]}(\cdot)\right| \\
& +\left|z_{n}+K_{1} z_{[n-1]}\right|\left|\widetilde{\phi}_{n}(\cdot)+K_{1} \widetilde{\phi}_{[n-1]}(\cdot)\right| .
\end{aligned}
$$

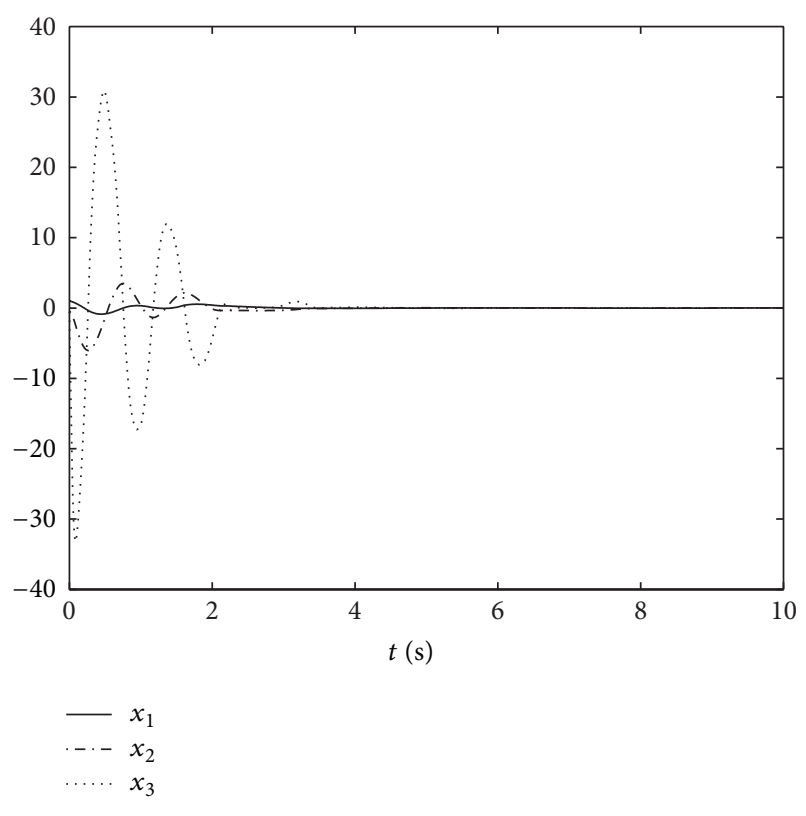

FIGURE 1: Trajectory of states $x_{1}, x_{2}$, and $x_{3}$.

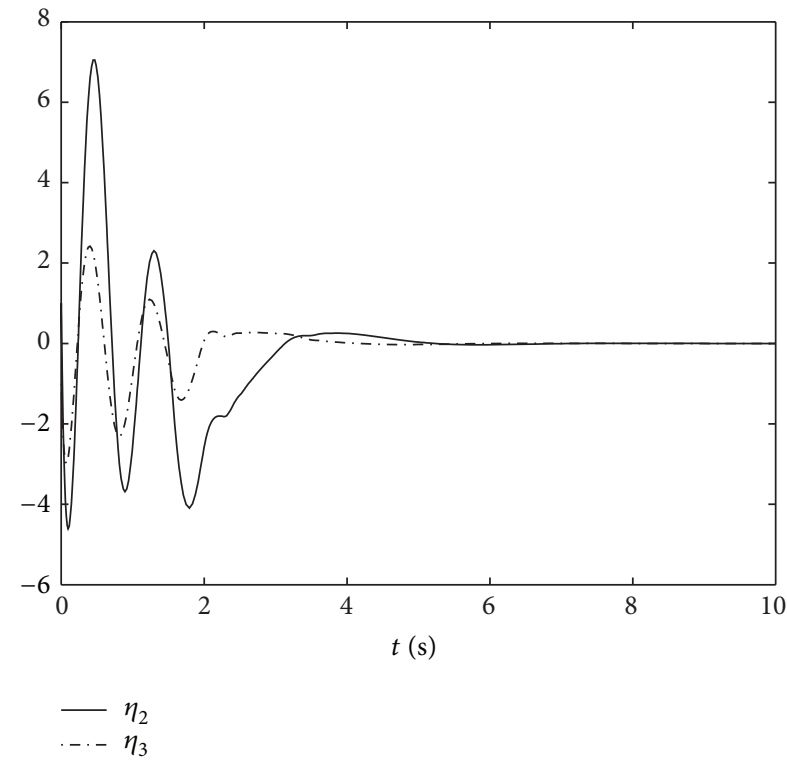

Figure 2: Trajectories of states $\eta_{2}$ and $\eta_{3}$.

In what follows, we estimate the right-hand side of (A.1). By $\left\|K_{1}\right\|^{2}\left\|z_{[n-1]}\right\|^{2} \geq\left(K_{1} z_{[n-1]}\right)^{2}$, we obtain

$$
\begin{aligned}
& \left\|z_{[n-1]}\right\|^{2}+\left(z_{n}+K_{1} z_{[n-1]}\right)^{2} \\
& \quad \geq \frac{1}{2}\left\|z_{[n-1]}\right\|^{2}+\frac{1}{2\left\|K_{1}\right\|^{2}}\left(K_{1} z_{[n-1]}\right)^{2}+\left(z_{n}+K_{1} z_{[n-1]}\right)^{2} \\
& \quad \geq \frac{1}{2}\left\|z_{[n-1]}\right\|^{2}+\frac{1}{2+4\left\|K_{1}\right\|^{2}} z_{n}^{2} \\
& \quad \geq c\|z\|^{2},
\end{aligned}
$$

where $c=1 /\left(2+4\left\|K_{1}\right\|^{2}\right)$. 


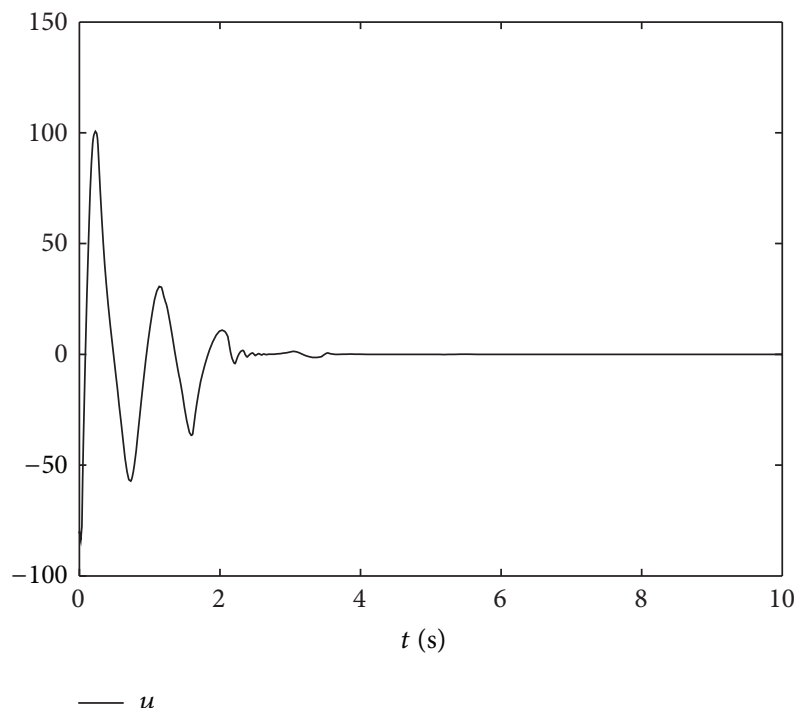

FIgURE 3: Trajectories of control $u$.

By the definitions of $z_{i}$ 's and $e_{i}$ 's, we have

$$
z_{i}-\widehat{z}_{i}=e_{i}+\ell_{i}\left(z_{i-1}-\widehat{z}_{i-1}\right), \quad i=2, \ldots, n,
$$

which implies

$$
\begin{gathered}
\left|z_{2}-\widehat{z}_{2}\right|=\left|e_{2}\right|, \\
\left|z_{i}-\widehat{z}_{i}\right| \leq\left|e_{i}\right|+\ell_{i}\left|e_{i-1}\right|+\cdots+\ell_{i} \cdots \ell_{3}\left|e_{2}\right|, \quad i=3, \ldots, n .
\end{gathered}
$$

Using (10) and (21) yields

$$
|v(\widehat{z})-\widetilde{v}(z)| \leq \frac{k_{n} \bar{k}}{g_{0}\left(z_{1}\right)} \sum_{i=2}^{n}\left|z_{i}-\mathbf{s a t}\left(\widehat{z}_{i}\right)\right|,
$$

where $\bar{k}=\max \left\{1, k_{1}, \ldots, k_{n-1}\right\}$. By this and (A.4) and noting that, for any $i=2, \ldots, n,\left|z_{i}-\operatorname{sat}\left(\widehat{z}_{i}\right)\right| \leq \min \left\{z_{i}-\widehat{z}_{i}, 2 M\right\}$ on $\Gamma_{M}^{z} \times \mathbf{R}^{n-1}$, we can find a positive constant $\lambda_{1}>1$ such that, on $\Gamma_{M}^{z} \times \mathbf{R}^{n-1}$,

$$
\begin{aligned}
& \mid v(\widehat{z})-\widetilde{v}(z) \|_{\Gamma_{M}^{z} \times \mathbf{R}^{n-1}} \\
& \quad \leq \frac{k_{n} \bar{k} \lambda_{1}}{g_{0}\left(z_{1}\right)} \min \left\{\left|e_{n}\right|+\sum_{i=2}^{n-1} \gamma_{i}\left(\ell_{i+1}, \ldots, \ell_{n}\right)\left|e_{i}\right|, 1\right\},
\end{aligned}
$$

where $\gamma_{i}\left(\ell_{i+1}, \ldots, \ell_{n}\right)=1+\ell_{i+1}+\sum_{j=i+2}^{n}\left(\ell_{i+1} \cdots \ell_{j}\right), i=2$, $\ldots, n-1$. Then, by Assumption 1, we derive that, on $\Gamma_{M}^{z} \times \mathbf{R}^{n-1}$,

$$
\begin{gathered}
\widetilde{g}(t, z)\left|z_{n}+K_{1} z_{[n-1]}\right||v(\widehat{z})-\widetilde{v}(z)| \\
\leq \bar{k} g_{1}\left(z_{1}\right) \sum_{i=1}^{n}\left|z_{i}\right||v(\widehat{z})-\widetilde{v}(z)| \\
\leq \frac{c}{2}\|z\|^{2}+\min \left\{W_{e}(e), \widehat{\alpha}_{n}\right\},
\end{gathered}
$$

where $\widehat{\alpha}_{i}\left(\ell_{i+1}, \ldots, \ell_{n}\right)=\left(n \bar{k}^{4} \lambda_{1}^{2} \lambda_{2}^{2} / 2 c\right) \gamma_{i}^{2}\left(\ell_{i+1}, \ldots, \ell_{n}\right), i=2$, $\ldots, n-1$, and $\widehat{\alpha}_{n}=n \bar{k} \lambda_{1}^{2} \lambda_{2}^{2} / 2 c$ with $\lambda_{2}=\max _{z \in \Gamma_{M}^{z}}\left(k_{n} g_{1}\left(z_{1}\right) /\right.$ $\left.g_{0}\left(z_{1}\right)\right)$.

Moreover, by (8), there exists a positive constant $d_{1}$ independent of $\ell_{i}$ 's, such that, on $\Gamma_{M}^{z} \times \mathbf{R}^{n-1}$,

$$
\begin{aligned}
& \left|z_{[n-1]}^{\mathrm{T}} P_{1} \widetilde{\phi}_{[n-1]}(\cdot)\right| \\
& +\left|z_{n}+K_{1} z_{[n-1]}\right| \mid \tilde{\phi}_{n}(\cdot)+K_{1} \tilde{\phi}_{[n-1]}(\cdot) \|_{\Gamma_{M}^{z} \times \mathbf{R}^{n-1}} \\
& \leq L^{\sigma} d_{1}\|z\|^{2} .
\end{aligned}
$$

By substituting (A.2), (A.7), and (A.8) into (A.1), we conclude that Proposition 4 holds.

\section{B. The Proof of Proposition 5}

By system (24), we have

$$
\begin{aligned}
\dot{V}_{e}(e) \leq & -L \sum_{i=2}^{n} e_{i} e_{i}^{2}+L \sum_{i=2}^{n-1}\left|e_{i}\right|\left|z_{i+1}\right| \\
& +L \sum_{i=2}^{n} \ell_{i}^{2}\left|e_{i}\right|\left|z_{i-1}-\widehat{z}_{i-1}\right| \\
& +L \widetilde{g}(t, z)\left|e_{n} v(\widehat{z})\right|+\sum_{i=2}^{n}\left|e_{i}\right|\left|\widetilde{\phi}_{i}(\cdot)-\ell_{i} \widetilde{\phi}_{i-1}(\cdot)\right| .
\end{aligned}
$$

By (A.4) and Young's inequality, the second and third terms in the right-hand side of (B.1) satisfy

$$
\begin{aligned}
& \sum_{i=2}^{n-1}\left|e_{i}\right|\left|z_{i+1}\right| \leq \frac{c}{8} \sum_{i=2}^{n-1} z_{i+1}^{2}+\frac{2}{c} \sum_{i=2}^{n-1} e_{i}^{2} \leq \frac{c}{8}\|z\|^{2}+\frac{2}{c} \sum_{i=2}^{n-1} e_{i}^{2}, \\
& \sum_{i=2}^{n} \ell_{i}^{2}\left|e_{i}\right|\left|z_{i-1}-\widehat{z}_{i-1}\right| \\
& \quad \leq \sum_{i=2}^{n} \ell_{i}^{2}\left|e_{i}\right|\left(\left|e_{i-1}\right|+\ell_{i-1}\left|e_{i-2}\right|+\cdots+\ell_{i-1} \cdots \ell_{3}\left|e_{2}\right|\right) \\
& \quad \leq \widetilde{\beta}_{n} e_{n}^{2}+\sum_{i=2}^{n-1} \widetilde{\beta}_{i}\left(\ell_{i+1}, \ldots, \ell_{n}\right) e_{i}^{2},
\end{aligned}
$$

where $\widetilde{\beta}_{n}=n-2$ and $\widetilde{\beta}_{i}\left(\ell_{i+1}, \ldots, \ell_{n}\right)=i-2+(1 / 4) \ell_{i+1}^{4}+$ $(1 / 4) \sum_{j=i+2}^{n}\left(\ell_{i+1}^{2} \cdots \ell_{j-1}^{2} \ell_{j}^{4}\right), i=2, \ldots, n-1$.

By (A.3) and (A.4), we have that

$$
\begin{gathered}
\left|\hat{z}_{2}\right| \leq\left|z_{2}\right|+\left|e_{2}\right|, \\
\left|\widehat{z}_{i}\right| \leq\left|z_{i}\right|+\left|e_{i}\right|+\ell_{i}\left|e_{i-1}\right|+\cdots+\ell_{i} \cdots \ell_{3}\left|e_{2}\right|, \quad i=3, \ldots, n,
\end{gathered}
$$


which, together with Young's inequality and Assumption 1, implies

$$
\begin{aligned}
\widetilde{g}(t, z)\left|e_{n} v(\widehat{z})\right|_{\Gamma_{M}^{z} \times \mathbf{R}^{n-1}} \\
\leq \frac{k_{n} g_{1}\left(z_{1}\right)}{g_{0}\left(z_{1}\right)}\left|e_{n}\right| \\
\quad \cdot\left|\operatorname{sat}\left(\widehat{z}_{n}\right)+k_{n-1} \operatorname{sat}\left(\widehat{z}_{n-1}\right)+\cdots+k_{2} \operatorname{sat}\left(\widehat{z}_{2}\right)+k_{1} \widehat{z}_{1}\right| \\
\leq \lambda_{2} \bar{k}\left|e_{n}\right| \sum_{i=1}^{n}\left|\widehat{z}_{i}\right| \\
\leq \frac{c}{8}\|z\|^{2}+\widehat{\beta}_{n} e_{n}^{2}+\sum_{i=2}^{n-1} \widehat{\beta}_{i}\left(\ell_{i+1}, \ldots, \ell_{n}\right) e_{i}^{2},
\end{aligned}
$$

where $\lambda_{2}=\max _{z \in \Gamma_{M}^{z}}\left(k_{n} g_{1}\left(z_{1}\right) / g_{0}\left(z_{1}\right)\right), \bar{k}=\max \left\{1, k_{1}, \ldots\right.$, $\left.k_{n-1}\right\}, \widehat{\beta}_{n}=n(n-1) \bar{k} \lambda_{2} / 2+2 \bar{k}^{2} \lambda_{2}^{2} / c$, and $\widehat{\beta}_{i}\left(\ell_{i+1}, \ldots, \ell_{n}\right)=$ $2 \bar{k}^{2} \lambda_{2}^{2} / c+\ell_{i+1}^{2}+\sum_{j=i+2}^{n}\left(\ell_{i+1}^{2} \cdots \ell_{j}^{2}\right), i=2, \ldots, n-1$.

Substituting (B.2) and (B.4) into (B.1), we conclude that (26) holds with $\widetilde{\alpha}_{n}=\widehat{\beta}_{n}+\widetilde{\beta}_{n}$, and $\widetilde{\alpha}_{i}\left(\ell_{i+1}, \ldots, \ell_{n}\right)=\widehat{\beta}_{i}\left(\ell_{i+1}\right.$, $\left.\ldots, \ell_{n}\right)+\widetilde{\beta}_{i}\left(\ell_{i+1}, \ldots, \ell_{n}\right), i=2, \ldots, n-1$.

\section{Conflict of Interests}

The authors declare that there is no conflict of interests regarding the publication of this paper.

\section{Acknowledgments}

This work was supported by the National Natural Science Foundations of China (61325016, 61273084, and 61233014), the National Science Foundation of Distinguished Young Scholar of Shandong Province of China (JQ200919), and the Independent Innovation Foundation of Shandong University of China (2012JC014).

\section{References}

[1] R. Sepulchre, "Slow peaking and low-gain designs for global stabilization of nonlinear systems," IEEE Transactions on Automatic Control, vol. 45, no. 3, pp. 453-461, 2000.

[2] S. Battilotti, "Generalized dilations and the stabilization of uncertain systems via measurement feedback," Systems \& Control Letters, vol. 43, no. 2, pp. 95-100, 2001.

[3] F. Esfandiari and H. K. Khalil, "Output feedback stabilization of fully linearizable systems," International Journal of Control, vol. 56, no. 5, pp. 1007-1037, 1992.

[4] H. K. Khalil and F. Esfandiari, "Semiglobal stabilization of a class of nonlinear systems using output feedback," IEEE Transactions on Automatic Control, vol. 38, no. 9, pp. 1412-1415, 1993.

[5] A. Teel and L. Praly, "Global stabilizability and observability imply semi-global stabilizability by output feedback," Systems and Control Letters, vol. 22, no. 5, pp. 313-325, 1994.
[6] A. Teel and L. Praly, "Tools for semiglobal stabilization by partial state and output feedback," SIAM Journal on Control and Optimization, vol. 33, no. 5, pp. 1443-1488, 1995.

[7] C. Qian, "Semi-global stabilization of a class of uncertain nonlinear systems by linear output feedback," IEEE Transactions on Circuits and Systems II: Express Briefs, vol. 52, no. 4, pp. 218222, 2005.

[8] B. Yang and W. Lin, "Semi-global stabilization of nonlinear systems by nonsmooth output feedback," International Journal of Robust and Nonlinear Control, vol. 24, no. 16, pp. 2522-2545, 2014.

[9] C. Zhang, R. Jia, C. Qian, and S. Li, "Semi-global stabilization via linear sampled-data output feedback for a class of uncertain nonlinear systems," International Journal of Robust and Nonlinear Control, 2014.

[10] M.-L. Liu and Y.-G. Liu, "Semiglobal stabilization via outputfeedback for a class of uncertain nonlinear systems," Acta Automatica Sinica, vol. 39, no. 12, pp. 2154-2159, 2013.

[11] R. Jia, C. Qian, and J. Zhai, "Semi-global stabilisation of uncertain non-linear systems by homogeneous output feedback controllers," IET Control Theory and Applications, vol. 6, no. 1, pp. 165-172, 2012.

[12] J.-Y. Zhai, W.-T. Zha, and S.-M. Fei, "Semi-global finite-time output feedback stabilization for a class of large-scale uncertain nonlinear systems," Communications in Nonlinear Science and Numerical Simulation, vol. 18, no. 11, pp. 3181-3189, 2013.

[13] B. Yang and W. Lin, "On semi-global stabilizability of MIMO nonlinear systems by output feedback," Automatica, vol. 42, no. 6, pp. 1049-1054, 2006.

[14] P. Krishnamurthy, F. Khorrami, and R. S. Chandra, "Global high-gain-based observer and back-stepping controller for generalized output-feedback canonical form," IEEE Transactions on Automatic Control, vol. 48, no. 12, pp. 2277-2284, 2003.

[15] P. Krishnamurthy and F. Khorrami, "High-gain outputfeedback control for nonlinear systems based on multiple time scaling," Systems \& Control Letters, vol. 56, no. 1, pp. 7-15, 2007.

[16] P. Krishnamurthy and F. Khorrami, "Dual high-gain-based adaptive output-feedback control for a class of nonlinear systems," International Journal of Adaptive Control and Signal Processing, vol. 22, no. 1, pp. 23-42, 2008.

[17] C. Qian and W. Lin, "Output feedback control of a class of nonlinear systems: a nonseparation principle paradigm," IEEE Transactions on Automatic Control, vol. 47, no. 10, pp. 1710-1715, 2002.

[18] H. Shim, I. Y. Son, and H. J. Seo, "Semi-global observer for multi-output nonlinear systems," Systems \& Control Letters, vol. 42, no. 3, pp. 233-244, 2001.

[19] C. Qian and W. Lin, "Recursive observer design, homogeneous approximation, and nonsmooth output feedback stabilization of nonlinear systems," IEEE Transactions on Automatic Control, vol. 51, no. 9, pp. 1457-1471, 2006. 


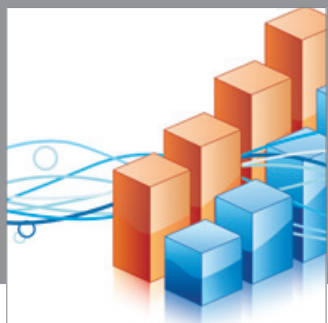

Advances in

Operations Research

mansans

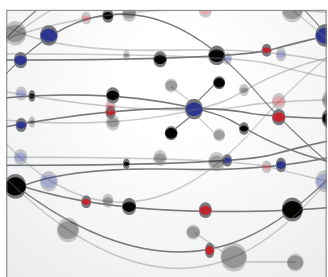

The Scientific World Journal
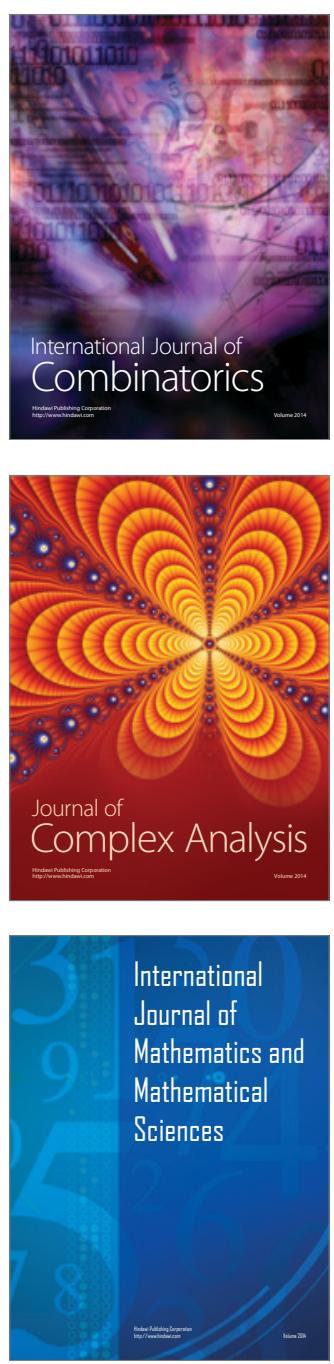
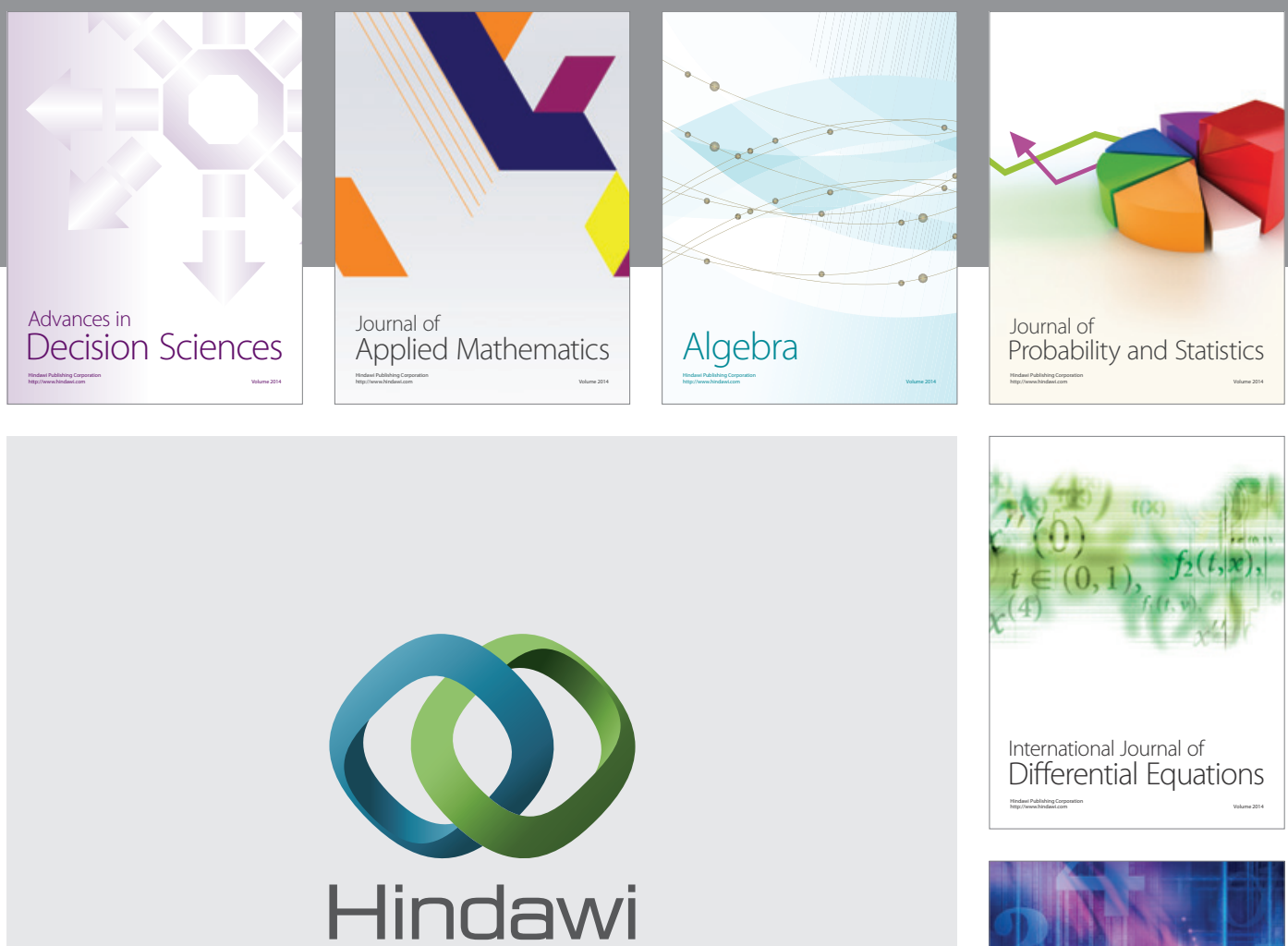

Submit your manuscripts at http://www.hindawi.com
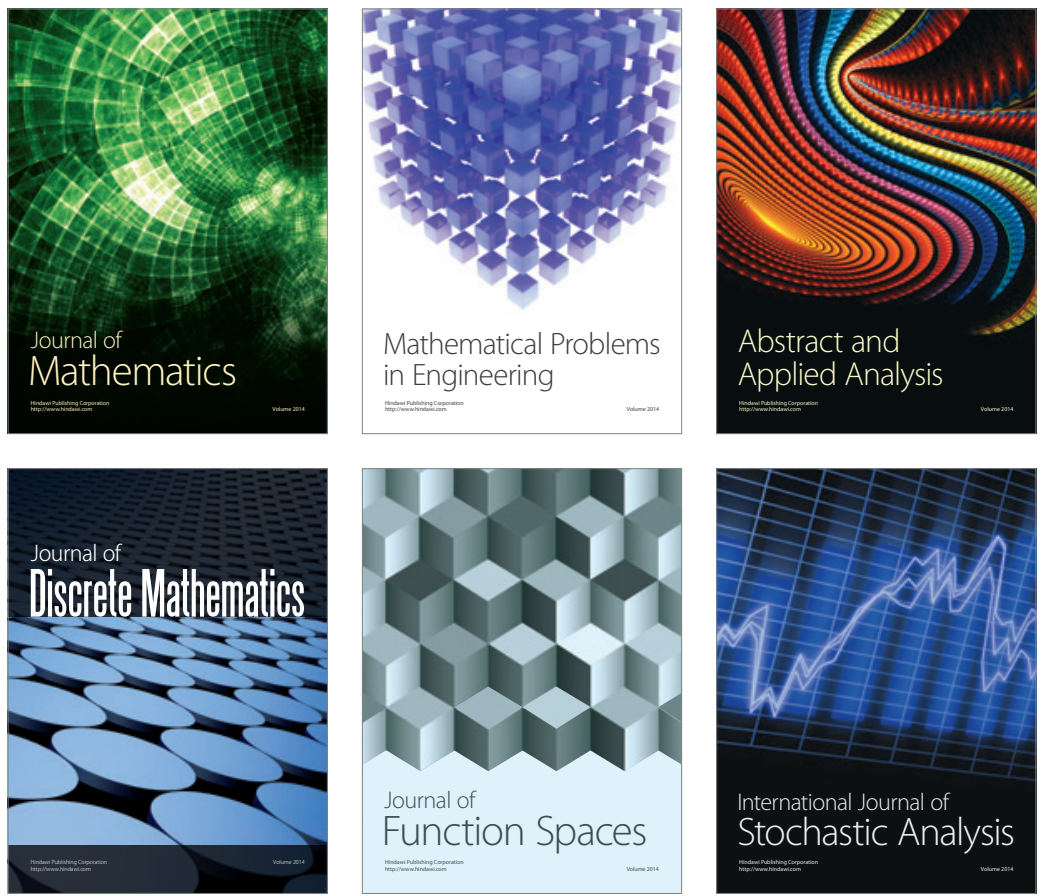

Journal of

Function Spaces

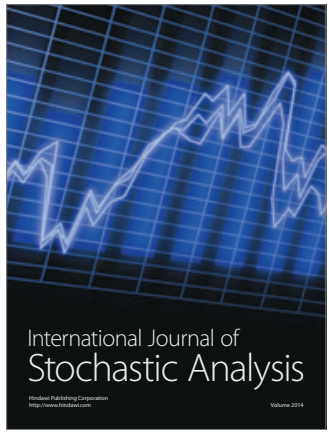

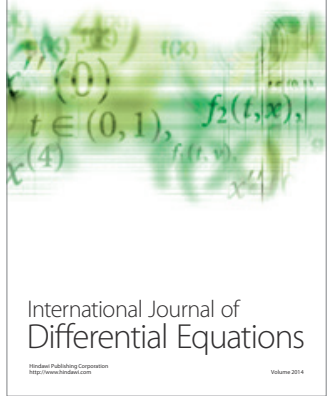
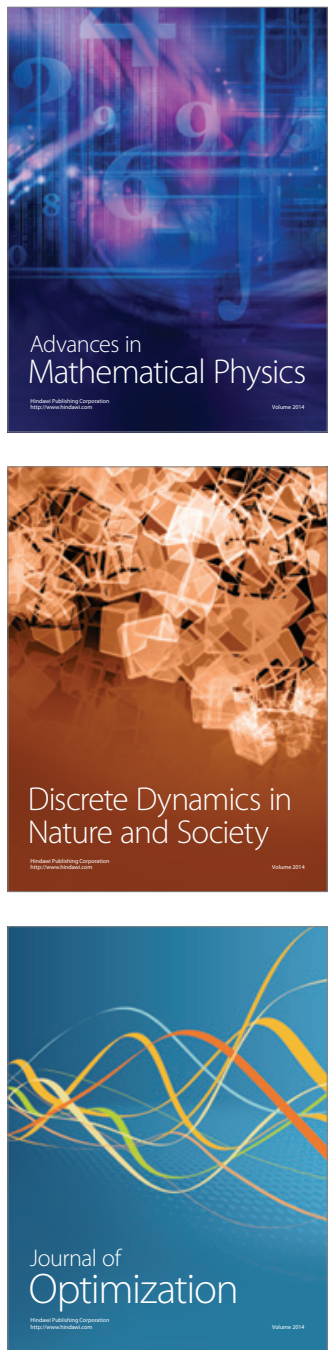